\title{
Learning to Recognize Names Across Languages
}

\author{
Anthony F. Gallippi \\ University of Southern California \\ University Park, EEB 234 \\ Los Angeles, CA 90089 \\ USA \\ gallippi@aludra.usc.edu
}

\begin{abstract}
The development of natural language processing (NLP) systems that perform machine translation (MT) and information retrieval (IR) has highlighted the need for the automatic recognition of proper names. While various name recognizers have been developed, they suffer from being too limited; some only recognize one name class, and all are language specific. This work develops an approach to multilingual name recognition that allows a system optimized for one language to be ported to another with little additional effort and resources. An initial core set of linguistic features, useful for name recognition in most languages, is identified. When porting to a new language, these features need to be converted (partly by hand, partly by on-line lists), after which point machine learning (ML) techniques build decision trees that map features to name classes. A system initially optimized for English has been successfully ported to Spanish and Japanese. Only a few days of human effort for each new language results in performance levels comparable to that of the best current English systems.
\end{abstract}

\section{Introduction}

Proper names represent a unique challenge for $\mathrm{M}^{\mathrm{T}} \mathrm{T}$ and IR systems. They are not found in dictionaries, are very large in number, come and go every day, and appear in many alias forms. For these reasons, list based matching schemes do not achieve desired performance levels. Hand coded heuristics can be developed to achieve high accuracy, however this approach lacks portability. Much human effort is needed to port the system to a new domain.

A desirable approach is one that maximizes reuse and minimizes human effort. This paper presents an approach to proper name recognition that uses ma- chine learning and a language independent framework. Knowledge incorporated into the framework is based on a set of measurable linguistic characteristics, or features. Some of this knowledge is constant across languages. The rest can be generated automatically through machine learning techniques.

The problem being considered is that of segmenting natural language text into lexical units, and of tagging those units with various syntactic and semantic features. A lexical unit may be a word (e.g., "started") or a phrase (e.g., "The Washington Post"). The particular lexical units of interest here are proper names. Segmenting and tagging proper names is very important for natural language processing, particularly IR and MT.

Whether a phrase is a proper name, and what type of proper name it is (company name, location name, person name, date, other) depends on (1) the internal structure of the phrase, and (2) the surrounding context.

Internal: "Mr. Brandon"

Context: "The new company, Safetek, will make air bags."

The person title "Mr." reliably shows "Mr. Brandon" to be a person name. "Safetek" can be recognized as a company name by utilizing the preceding contextual phrase and appositive "The new company,".

The recognition task can be broken down into delimitation and classification. Delimitation is the determination of the boundarics of the proper name, while classification serves to provide a more specific category.

Original: John Smith, chairman of Safetek, announced his resignation yesterday.

Delimit: $\langle\mathrm{PN}\rangle$ John Smith $\langle/ \mathrm{PN}\rangle$, chairman of $<\mathrm{PN}>$ Safetek $<\mathrm{PN}>$, announced his resignation yesterday.

Classify: <person $>$ John Smith </person $>$, chairman of <company $>$ Safetek </company $>$, announced his resignation yesterday. 
During the delimit step, the boundaries of all proper names are identified. Next, the delimited proper names are classilied into more specific categorics.

How can a system developed in one language be ported to another language with minimal additional effort and comparable performance results? How much additional effort will be required, and what degradation in performance, if any, is to be expected" These questions are addressed in the following sections.

\section{Method}

The approach taken here is to utilize a data-driven knowledge acquisition strategy based on decision trees which uses contextual information. This differs from other approaches which attempt to achicve this task by: (1) hand-coded heuristics, (2) list-based matching schemes, (3) human-gencrated knowledge bases, and (4) combinations thereof. Delimitation occurs through the application of phrasal templates. These templates, built by hand, use logical operators (AND, OR, etc.) to combine features strongly associated with proper names, including: proper noun, ampersand, hyphen, and comma. In addition, ambiguitics with delimitation are handled by including other predictive features within the templates.

T'o acquire the knowledge required for classification, each word is tagged with all of its associated features. These features are obtained through automated and manual techniques. A decision tree is built (for each name class) from the initial feature set using a recursive partitioning algorithm (Quinlan, 1986; Breiman et al., 1984) that uses the following function as its selection (splitting) criterion:

$$
-p^{*} \log 2(p)-(1-p)^{*} \log 2(1-p)
$$

where $p$ represents the proportion of names belonging to the class for which the tree is built. The foature which minimizes the weighted sum of this function across both child nodes resulting from the split is chosen. A multitree approach was chosen over learning a single tree for all name classes because it allows for the straightforward association of features within the tree with specific name classes, and facilitates troubleshooting.

the result is a hicrarchical collection of co-occurring features which predict inclusion to or exclusion from a particular proper name class. Since a tree is built for each name class of interest, the trees are all applied individually, and then the results are merged.

\section{1 lieatures}

Various types of features indicate the type of name: parts of specch, designators, morphology, syntax, semantics, and more. Designators are features which alone provide strong evidence for or against a particular name type. Iixamples include "Co." (company), "Dr." (person), and "County" (location), lor example, of all the company names in the English training text, $28 \%$ are associated with a corporate designator.

Other fealures are predetermined, obtained viat online lists, or are selected automatically hased on statistical measures. Parts of speech features are predetermined based on the part of speech tagger employed. On-line lists provide lists of eities, person names, nationalities, regions, etc. The initial set of lexical features is selected by choosing those that appear most frequently (above some threshold) throughout the training data, and those that appear most frequently near the positive instances in the training data.

Some features, such as morphological, keyword, and key phrase features, are determined by hand analysis of the text. Capitalization is one obvious

Table 1. Features summary.

\begin{tabular}{|c|c|c|c|}
\hline Type & Feature & Iixample & How many \\
\hline Pat of Speech & $\begin{array}{l}\text { Proper Noum } \\
\text { Common Noun }\end{array}$ & $\begin{array}{l}\text { "Aristotle" } \\
\text { "philosophy" }\end{array}$ & $\begin{array}{l}\text { NA } \\
\text { NA }\end{array}$ \\
\hline Designitur & $\begin{array}{l}\text { Company } \\
\text { Person } \\
\text { Location } \\
\text { Date }\end{array}$ & $\begin{array}{l}\text { "Coty.", "Ltd!" } \\
\text { "Mr.", "President" } \\
\text { Country, State, City } \\
\text { Month, I Day of weck }\end{array}$ & $\begin{array}{l}100 \mathrm{E}, 110 \mathrm{~S}, 60 \mathrm{~J} \\
70 \mathrm{E}, 70 \mathrm{~S}, 43 \mathrm{~J} \\
520 \mathrm{E}, 900 \mathrm{~S}, 570 \mathrm{~J} \\
56 \mathrm{E}, 19 \mathrm{~S}, 19 \mathrm{~J} \\
\end{array}$ \\
\hline Morphology & $\begin{array}{l}\text { Capitalization } \\
\text { Company Sullix } \\
\text { Word Length }\end{array}$ & $\begin{array}{l}\text { "A.", "13-" } \\
\text { "-cori"," "-tec" } \\
\text { WL }>8, \text { WL }<3\end{array}$ & $\begin{array}{l}1 \mathrm{E}, 1 \mathrm{~S}, 0 \mathrm{~J} \\
5 \mathrm{E}, 0 \mathrm{~S}, 30 \mathrm{~J} \\
4 \mathrm{E}, 4 \mathrm{~S}, 2 \mathrm{~J} \\
\end{array}$ \\
\hline list & $\begin{array}{l}\text { Companies } \\
\text { Persons } \\
\text { Locations } \\
\text { Nationalities } \\
\text { Keyword(s) } \\
\end{array}$ & $\begin{array}{l}\text { "IBM", "AT\&'1" } \\
\text { "Smith", "Michael" } \\
\text { "Ciulf of Mexico" } \\
\text { "Japanese" } \\
\text { "based in", "said he" }\end{array}$ & $\begin{array}{l}0 \mathrm{E}, 100 \mathrm{~S}, 7 \mathrm{~K} \mathrm{~J} \\
2 І \mathrm{~K} \mathrm{~L}, 2 \perp \mathrm{K} \mathrm{S}, 185 \mathrm{~K} \mathrm{~J} \\
20 \mathrm{E}, 20 \mathrm{~S}, 2 \mathrm{~K} \mathrm{~J} \\
220 \mathrm{H}, 0 \mathrm{~S}, 0 \mathrm{~J} \\
44 \mathrm{E}, 49 \mathrm{~S}, 54 \mathrm{~J}\end{array}$ \\
\hline Template & $\begin{array}{l}\text { Company } \\
\text { Person } \\
\text { Location } \\
\text { Date } \\
\text { Proper Name } \\
\end{array}$ & $\begin{array}{l}<\mathrm{NNP} \text { CN desig }> \\
<\mathrm{P}^{\mathrm{l}} \text { lesig NNP }> \\
<\mathrm{NNl} \mathrm{L} \text { desig }> \\
<\mathrm{MMNum}, \mathrm{Num}> \\
<\mathrm{NNI} \text { NNP }>\end{array}$ & $\begin{array}{l}210 \mathrm{E}, 210 \mathrm{~S}, 210 \mathrm{~J} \\
90 \mathrm{E}, 95 \mathrm{~S}, 90 \mathrm{~J} \\
190 \mathrm{E}, 190 \mathrm{~S}, 190 \mathrm{~J} \\
17 \mathrm{E}, 18 \mathrm{~S}, 70 \mathrm{~J} \\
140 \mathrm{E}, 140 \mathrm{~S}, 140 \mathrm{~J}\end{array}$ \\
\hline Special Purpose & $\begin{array}{l}\text { Dungst Com Sbstr } \\
\text { Duplicated PNs }\end{array}$ & $\begin{array}{l}\text { "VW" <- Volkswagen } \\
\text { DUP 2t, DUP 5t }\end{array}$ & $\begin{array}{l}1 \mathrm{IJ}, 1 \mathrm{~S}, 1 \mathrm{~J} \\
5 \mathrm{E}, 5 \mathrm{~S}, 2 \mathrm{~J}\end{array}$ \\
\hline
\end{tabular}


morphological feature of importance. Determining keyword and key phrase features amounts to selecting prudent subject categories. These categories are associated with lists of lexical items or already existing features. For example, many of the statistically derived lexical features may fall under common subject categories. The words "build", "make", "manufacture", and "produce" can be associated with the subject category "make-type verbs". Analysis of the immediate context surrounding company names may lead to the discovery of key phrases like "said it", "entered a venture", and "is located in". Table 1 shows a summary of various types of features used in system development. The longest common substring (LCS) feature (Jacobs et $a l$,, 1993) is useful for finding proper name aliases.

\subsection{Feature Trees}

The ID3 algorithm (Quinlan, 1986) selects and organizes features into a discrimination tree, one tree for each type of name (person, company, ctc.). The tree, once built, typically contains $100+$ nodes, each one inquiring about one feature in the text, within the locality of the current proper name of interest.

An example of a tree which was generated for companies is shown in Figure 1. The context level for this example is 3, meaning that the feature in question must occur within the region starting 3 words to the left of and ending 3 words to the right of the proper name's left boundary. A "(L)" or "(R)" following the feature name indicates that the feature must occur to the left of or to the right of the proper name's left boundary respectively. The numbers directly beneath a node of the tree represent the number of negative and positive examples present from the training set. These numbers are useful for associating a confidence level with each classification. Definitions for the features in Figure 1 (and other abbreviations) can be found in the appendix.

The training set used for this example contains 1084 negative and 669 positive examples. To obtain the best initial split of the training set, the feature "CN_alias" is chosen. Recursively visiting and optimally splitting each concurrent subset results in the generation of 97 nodes (not including leaf nodes).

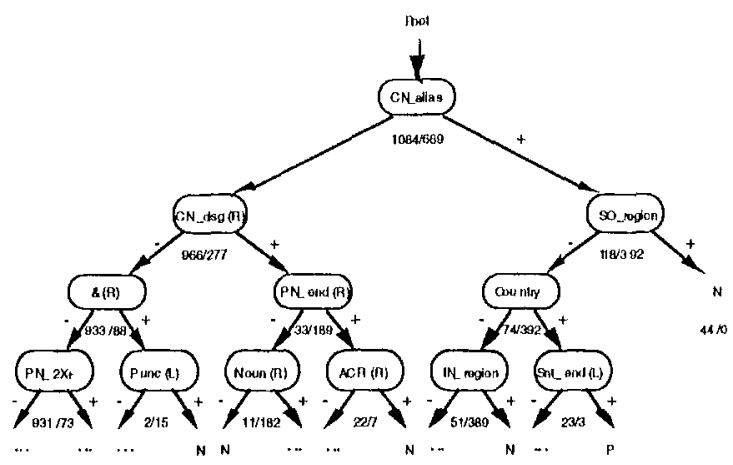

Figure 1. Company tree example (context is $+/-3$ ).

\subsection{Architecture}

Figure 2 shows the working development system. The starting point is training text which has been pretagged with the locations of all proper names. The tokenizer separates punctuation from words. For non-token languages (no spaces between words), it also separates contiguous characters into constituent words. The part of speech (POS) tagger (Brill, 1992; Farwell et. al., 1994; Matsumoto et al., 1992) attaches parts of speech. The set of derived features is attached. During the delimitation phase, proper names are delimited using a set of POS-based handcoded templates. Using ID3, a decision tree is generated based on the existing feature set and the specified level of context to be considered. The generated tree is applied to test data and scored. Manual analysis of the tree and scored result leads to the discovery of new features. The new features are added to the tokenized training text, and the process repeats.

\subsection{Cross Language Porting}

In order to work with another language, the following resources are needed: (1) pre-tagged training text in the new language using same tags as before, (2) a tokenizer for non-token languages, (3) a POS tagger (plus translation of the tags to a standard POS convention), and (4) translation of designators and lexical (list-based) features.

These language-specific modules are highlighted in Figure 2 with bold borders. Feature translation occurs through the utilization of: on-line resources, dictionaries, atlases, bilingual speakers, etc. The remainder is constant across languages: a language independent core development system, and an optimally derived feature set for English.

Also worth noting are the parts of development system that are cxecuted by hand. These are shown shaded. Everything else is automatic.

\section{Experiment}

The system was first built for English and then ported to Spanish and Japanese. For English, the training text consisted of 50 messages obtained from the English Joint Ventures (EJV) domain MUC-5 corpus of the US Advanced Research Projects Agency (ARPA). This data was hand-tagged with the locations of company names, person names, locations names, and dates. The test set consisted of 10 new messages.

Experimental results were obtained by applying the generated trees to test texts. The initial raw text is tokenized and tagged with parts of speech. All features necessary to apply rules and trees are attached. Phrasal template rules are applied in order to delimit proper names. Then trees for each proper name type are applied individually to the proper names in the featurized text. Proper names which are 


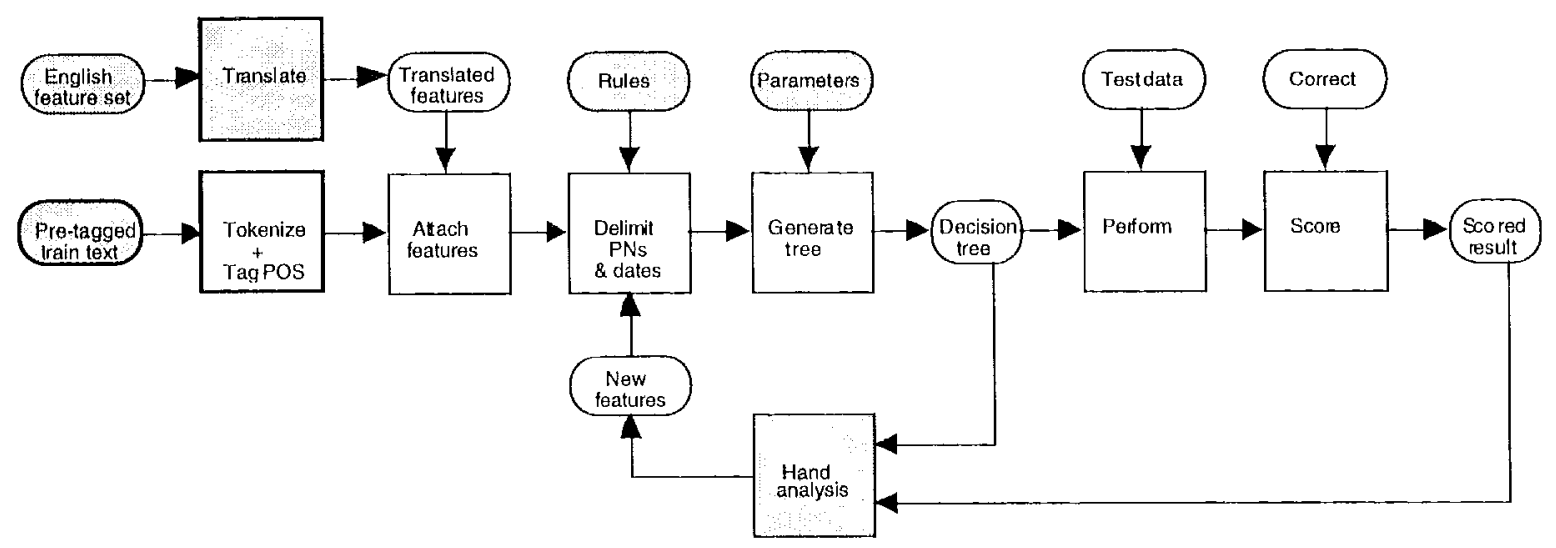

Figure 2. Multilingual development system.

voted into more than one class are handled by choosing the highest priority class. Priorities are determined based on the independent performance of each tree. For example, if person trees perform better independently than location trees, then a person classification will be chosen over a location classification. Also, designators have a large impact on resolving conflicts.

\subsection{English}

Various parameterizations were used for system development, including: (1) context depth, (2) feature set size, (3) training set size, and (4) incorporation of hand-coded phrasal templates.

Figure 3 shows the performance results for English. The metrics used were recall $(\mathrm{R})$, precision $(\mathrm{P})$, and an averaging measure, P\&R, defined as:

$$
\mathrm{P} \& \mathrm{R}=2 * \mathrm{P} * \mathrm{R} /(\mathrm{P}+\mathrm{R})
$$

Obtained results for English compare to the English results of Rau (1992) and McDonald (1993). The weighted average of the P\&R for companies, persons, locations, and dates is $94.0 \%$.

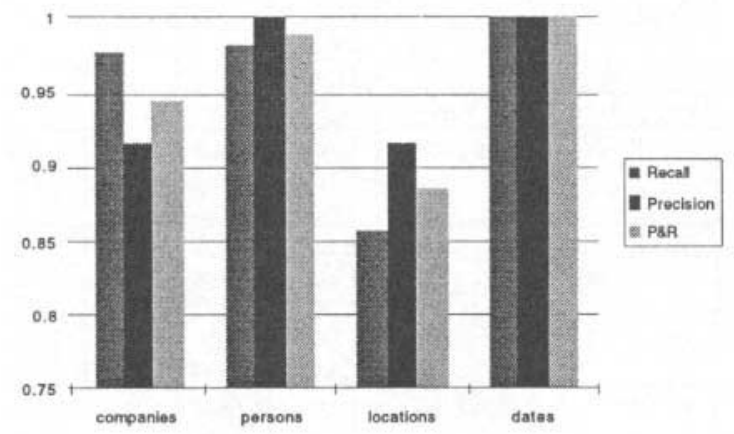

Figure 3. English performance results.

The date grammar is rather small in comparison to other name classes, hence the performance for dates was perfect. Locations, by contrast, exhibited the lowest performance. This can be attributed mainly to: (1) locations are commonly associated with commas, which can create ambiguitics with delimitation, and (2) locations made up a small percentage of all names in the training set, which could have resulted in overfitting of the built tree to the training data.

Features strengths were measured for companies, persons, and locations. This experiment involved removing one feature at a time from the text used for testing and then reapplying the same tree. Figure 4 and Table 2 show performance results (P\&R) when the three most powerful features are removed, one at a time, for companies, persons, and locations respectively. This experiment demonstrates the power of designator features across all proper name types, and the importance of the alias feature for companies.

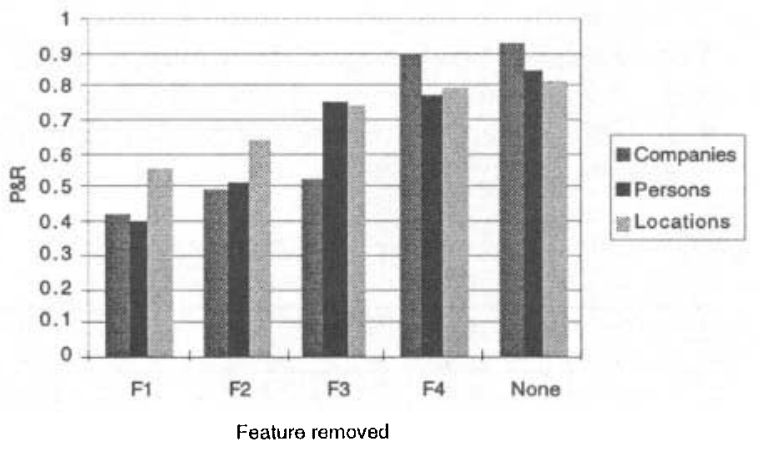

Figure 4. Feature strengths for English.

'Table 2. Strongest features for English.

\begin{tabular}{|l|l|l|l|}
\hline Feature & Companies & Persons & Locations \\
\hline F1 & CAP & P_desig & CAP \\
\hline F2 & CN_desig & CAP & L_desig \\
\hline F3 & CN_alias & ATH_reg & In \\
\hline F4 & Hyphen & F_I_L & Region \\
\hline
\end{tabular}

\subsection{Spanish}

Three experiments have been conducted for Spanish. In the first experiment, the English trees, generated 
from the feature set optimized for English, are applied to the Spanish text (E-E-S). In the second experiment, new Spanish-specific trees are generated from the feature set optimized for English and applied to the Spanish text (S-E-S). The third experiment proceeds like the second, except that minor adjustments and additions are made to the feature set with the goal of improving performance (S-S-S).

The additional resources required for the first Spanish experiment (E-E-S) are a Spanish POS-tagger (Farwell et al., 1994) and also the translated feature set (including POS) optimally derived for English. The second and third Spanish experiments (S-E-S, S-S-S) require in addition pre-tagged Spanish training text using the same tags as for English.

The obtained Spanish scores as compared to the scores from the initial English experiment (E-E-E) are shown in figure 5.

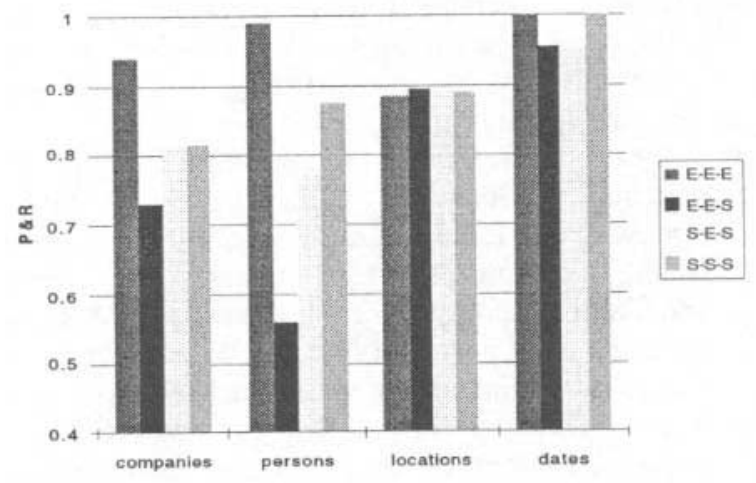

Figure 5. P\&R scores for Spanish versus English.

The additional Spanish specific features derived for S-S-S are shown in Table 3 . Only a few new features added to the core feature set allows for significant performance improvement.

Table 3. Spanish specific features for S-S-S.

\begin{tabular}{|c|c|c|c|}
\hline Type & Feature & Instances & $\begin{array}{l}\text { How } \\
\text { many }\end{array}$ \\
\hline List & $\begin{array}{l}\text { Companies } \\
\text { Keyword(s) }\end{array}$ & $\begin{array}{l}\text { "IBM", "AT\&T", ... } \\
\text { "(del" (OF THE) }\end{array}$ & $\begin{array}{l}100 \\
1 \\
\end{array}$ \\
\hline Template & $\begin{array}{l}\text { Person } \\
\text { Person } \\
\text { Date } \\
\text { Date }\end{array}$ & $\begin{array}{l}<\text { FN DE LN }> \\
<F N \text { DE NNP }> \\
<\text { Num OF MM }> \\
<\text { Num OF MM OF Num }>\end{array}$ & $\begin{array}{l}1 \\
1 \\
1 \\
1\end{array}$ \\
\hline
\end{tabular}

\subsection{Japanese}

The same three experiments conducted for Spanish are being conducted for Japanese. The first two, E-E-J and J-E-J, have been completed; J-J-J is in progress.

The additional resources required for the first Japanese experiment (E-E-J) are a Japanese tokenizer and POS-tagger (Matsumoto et al., 1992) and also the translated feature set optimally derived for English. The second and third Japanese experiments
$(\mathrm{J}-\mathrm{E}-\mathrm{J}, \mathrm{J}-\mathrm{J}-\mathrm{J})$ require in addition pre-tagged Japanese training text using the same tags as for English.

The obtained Japanese scores as compared to the scores from the initial English experiment (E-E-E) are shown in Figure 6. The weighted averages of the P\&R measures across all languages, for companics, persons, locations, and dates, are shown in Figure 7. Table 4 shows comparisons to other work.

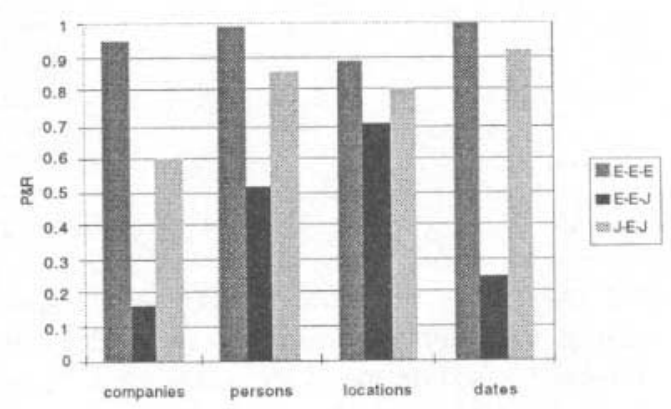

Figure 6. P\&R scores for Japanese versus English.

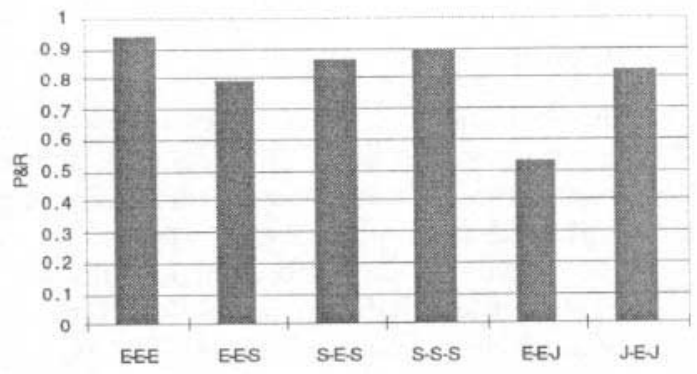

Figure 7. Weighted P\&R scores comparison.

Table 4. Performance comparison to other work.

\begin{tabular}{|l|l|l|l|l|l|}
\hline System & Lang. & Class & R & P & P\&R \\
\hline Rau & English & Com & NA & 95 & NA \\
\hline PNF & English & Com & NA & NA & "Ncar \\
(McDonald) & & Pers & & & $100 \%$ \\
& & Loc & & & \\
& & Date & & & \\
\hline Panglyzer & Spanish & NA & NA & 80 & NA \\
\hline MAJESTY & Japanese & Com & 84.3 & 81.4 & 82.8 \\
& & Pers & 93.1 & 98.6 & 95.8 \\
& & Loc & 92.6 & 96.8 & 94.7 \\
\hline MNR & English & Com & 97.6 & 91.6 & 94.5 \\
(Gallippi) & & Pers & 98.2 & 100 & 99.1 \\
& & Loc & 85.7 & 91.7 & 88.6 \\
& & Datc & 100 & 100 & 100 \\
& & (Avg) & & & 94.0 \\
\hline MNR & Spanish & Com & 74.1 & 90.9 & 81.6 \\
& & Pers & 97.4 & 79.2 & 87.4 \\
& & Loc & 93.1 & 87.5 & 89.4 \\
& & Date & 100 & 100 & 100 \\
& & (Avg) & & & 89.2 \\
\hline MNR & Japanese & Com & 60.0 & 60.0 & 60.0 \\
& & Pers & 86.5 & 84.9 & 85.7 \\
& & Loc & 80.4 & 82.1 & 81.3 \\
& & Date & 90.0 & 94.7 & 92.3 \\
& & (Avg & & & 83.1 \\
\hline
\end{tabular}




\section{Related Work}

Proper name recognition thas been addressed by others (Farwell et al., 1994; Kitani \& Mitamura, 1994; Rau, 1992), with the goal of incorporating this capability into IR and MT systems. Related problems have been studied which utilize contextual information and learning. Examples include postediting of documents (article selection) (Knight \& Chander, 1994), word sense disambiguation (Black, 1988; Sicgel \& McKeown, 1994), and discourso analysis (Soderland \& Lehnert, 1994).

\section{Future Work}

An investigation of the causes of perlormance degradation across languages will be conducted, with the goal of pinpointing and concurtently taking steps to minimize their elfects. Other plans include using MI. techniques to further reduce the amount of human effort: (1) automate the building of templates for delimitation, (2) automate the discovery of new features from test results, and (3) expand the search space traversed by the tree building algorithm to include splits on feature combinations.

\section{Acknowledgments}

The author would like to ofler special thanks and gratitude to liduard Hovy for all of his support, direction, and encouragement from the onset of this work. Thanks also to Kevin Knight for his carly suggestions, and to the Information Sciences Institute for use of their lacilities and resources.

\section{References}

Black, Ii. 1988. An Iixperiment in Computational Discrimination of English Word Senses. In IBM Journal of Research and Development, 32(2).

Breiman, I.., Vriedman, J.H., Olshen, R.A., and Stone, C.J. 1984. Classification and Regression Trees. Wadsworth Intcrnational Group.

Brill, 1:. 1992. ^ Simple Rule-Based Part of Specel Tagger. In Proceedings of the Third Conference on Applied Natural Language Processing, ACL.

Farwell, D., Helmreich, S., Jin, W., Casper, M., Hargrave, J., Molina-Salgado, H., and Weng, li. 1994. Panglyzer; Spanish I anguage Analysis System. In Proceedings of the Conference of the Association of Machine Translation in the Americas (ATMA). Columbia, MI).

Jacobs, P.S., Krupta, C., Rau, I.., Mauldin, M.I.. Mitamura, 'l', Kitani, '1., Sider, I. and Childs, L. 1993. GE-CMU: Description of the SHOGiUN System Used for MUC:-5. In Proceedings of the Fifth Message Understanding Conference (MUC-5). Morgan Kaufmann, p]. 109-120.

Kitani, T. and Milanmura, 'T, 1994. An Accurate Morphological Analysis and Proper Name Identifi. cation for Japanese Text Processing. In Transactions of Information Processing Socicty of Japan, Vol. 35, No. 3, pp. 404-413.

Knight, K. and Chander, I. 1994. Automated lostediting of Documents. In Proceedings of the Twelfth National Conference on Artificial Intelligence $(A A \Lambda I)$, pp. 779-784.

Lchncrt, W., McCarthy, J., Soderland, S., Riloff, E., Cardic, C., Peterson, J., Feng, F., Dolan, C., and Goldman, S. 1993. UMass/Hughes: Description of the CIRCUS System Used for MUC-5. In Proceedings of the Fifth Message Understanding Conference (MUC-5). Morgan Kaufinann, pp. 277-292.

Matsumoto, Y., Kurohashi, S., 'Taegi, H. and Nagao, M. 1992. JUMAN Users' Manual Version 0.8 , Nagao Laboratory, Kyoto University.

MeDonald, D. 1993. Internal and Lixternal Bividence in the Identification and Semantic Catcgorization of Proper Names. In Proceedings of the SINGLEX workshop on "Acquisition of lexical Knowledge from Text”, p1). 32-43.

Quinlan, J.R. 1986. Induction of Decision Trees. In Machine Learning, pp. 81-106.

Ratu, L.t. 1992. Extracting Company Names from 'lext. In Proceedings of the Seventh Confer"nce on Artificial Intelligence Applications, pp. 189194.

Sicgel, E.V. and McKoown, K.R. 1994. Jimergent Linguistic Rules from Inducing Decision Trees: Disambiguating Discourse Cluc Words. In Proceedings of the Twelfth National Conference on Artificial Intelligence (AAAI), pp. 820-826.

Soderland, S. and Ichnert, W. 1994. CorpusDriven Knowledge Acquisition for Discourse Analysis. In Proceedings of the Twelfth National Conference on Artificial Intelligence (AAAI), p. 827-832.

\section{Appendix A. Abbreviations}

Table 5. Definitions for abbreviations.

\begin{tabular}{|c|c|}
\hline Abloreviation & Defintition \\
\hline$\overline{A C R}$ & Acronym \\
\hline АТҢ $r e g$ & Occurs in $\langle\wedge$ uthor $>\ldots</ \Lambda$ uthor $>$ \\
\hline $\mathrm{CAl}^{\prime}$ & Capitalized \\
\hline CN alias & LCS of full company name \\
\hline CN_ding & Comprany name designator \\
\hline Country & Country name \\
\hline FN & First (given) name \\
\hline I I I & First natne + initial + last name \\
\hline Hyphen & Hyphen (punctuation) \\
\hline IN region & ()ecurs in $\langle\mathrm{IN}\rangle \ldots</ \mathrm{IN}\rangle$ icgion \\
\hline In & I cxical "in" \\
\hline I.CS & Longest common substring \\
\hline I,$N$ & Last (family) name \\
\hline L desigr & L,ocation designator \\
\hline NNP & Proper noun \\
\hline Noun & Gencral noum \\
\hline PN_cnd & l'roper name end delimiter \\
\hline$P N 2 X+$ & Proper mame occurs $2+$ times \\
\hline Punc & Punctuation \\
\hline P desiyg & Person designator \\
\hline Region & Geographical region natuc \\
\hline SO region & Occus in $<\mathrm{SO}>\ldots</ \mathrm{SO}>$ region \\
\hline Sut end & Sentence end boundary \\
\hline$\&$ & Ampersind character \\
\hline
\end{tabular}

